\title{
Sistemática en el Tratamiento Quirúrgico de las Fracturas Panfaciales Aplicado a un Caso Clínico de Alta Complejidad
}

\author{
Systematic in the Surgical Treatment of Panfacial \\ Fractures Applied to a Highly Complex Clinical Case
}

\author{
Marcelo Mardones M. ${ }^{1,3,4}$; Rodrigo Bravo A..,3,4; María de los Ángeles Fernández T.2,3,4 \\ Renato Gunckel M. ${ }^{1,3,4}$ \& Coral Torres M. ${ }^{1}$
}

\begin{abstract}
MARDONES, M. M.; BRAVO, R. A.; FERNÁNDEZ, T. M. Á.; GUNCKEL, M. R. \& TORRES, M. C. Sistemática en el tratamiento quirúrgico de las fracturas panfaciales aplicado a un caso clínico de alta complejidad. Int. J. Odontostomat., 14(4):590-595, 2020.
\end{abstract}

RESUMEN: Las fracturas panfaciales son aquellas que afectan de forma simultánea al tejido óseo de dos o más tercios del rostro. El manejo inicial de estos pacientes es resolver o estabilizar las urgencias médico - quirúrgicas que puedan presentar, debido a que la gran mayoría de estas fracturas están asociadas a traumatismos de alta energía cinética, las que determinan la presencia de lesiones concomitantes que pueden comprometer la vida del paciente. El tratamiento quirúrgico de las fracturas panfaciales puede abarcar desde la intervención quirúrgica inicial de control de daños, estabilización, reducción y fijación quirúrgica de los segmentos fracturados mediante osteosíntesis hasta intervenciones mediatas para la reconstrucción de los tejidos afectados por el traumatismo. Para el tratamiento de las fracturas panfaciales existe una sistemática quirúrgica que tiene por objeto contener y/o minimizar daños agudos y permitir una reducción morfológica y funcional. Reportamos un caso clínico de fractura panfacial cuyo tratamiento siguió esta sistemática terapeútica.

PALABRAS CLAVE: fractura panfacial, politraumatizado, manejo inicial del trauma.

\section{INTRODUCCIÓN}

Las fracturas panfaciales se definen como aquellas fracturas que afectan de forma simultánea a dos o más tercios del rostro (Yang et al., 2012). Suelen ser causadas por traumatismos de alta energía cinética (lesiones por accidentes de tránsito o traumatismos por impacto balístico) y representan entre el $4 \%$ y el $10 \%$ de todas las fracturas faciales (Kim et al., 2016). Por lo general ocurren en forma concomitante a otras lesiones que pueden comprometer la vía aérea del paciente o estar asociadas a lesiones intracraneales y de la columna cervical, de manera que es fundamental tener en consideración el manejo inicial del trauma: control de la vía aérea y columna cervical, respiración, circulación, valoración del estado neurológico y exposición y control ambiental (ABCDE) para la estabilización del paciente (Chukwulebe \& Hogrefe, 2019). Posterior a la estabilización del paciente, el cirujano maxilofacial es el encargado de dar tratamiento quirúrgico a las fracturas panfaciales, y para un tratamiento exitoso requiere de la comprensión de los componentes de los patrones de fractura y la capacidad de relacionarlos anatómicamente con los elementos estables del cráneo (Choi \& Kim, 2019). El enfoque de la reconstrucción facial debe centrarse en el restablecimiento de las relaciones tridimensionales adecuadas, así como en la restauración de los volúmenes de la cavidad orbitaria, oral y nasal (Rodrigues et al., 2017).

\footnotetext{
${ }^{1}$ Equipo Cirugía Maxilofacial, Servicio de Cirugía, Hospital San José, Santiago, Chile.

${ }^{2}$ Equipo de Cirugía Maxilofacial, Hospital San Juan de Dios, Santiago, Chile..

${ }^{3}$ Departamento de Cirugía y Traumatología Bucal y Maxilofacial, Facultad Odontología, Universidad de Chile, Santiago, Chile.

${ }^{4}$ Departamento de Cirugía Maxilofacial Clínica Las Condes, Santiago, Chile.
} 


\section{REPORTE DEL CASO}

Paciente, sexo masculino, 24 años, ingresa de urgencia por caída en bicicleta, politraumatizado y trauma en región facial y craneal. Es evaluado

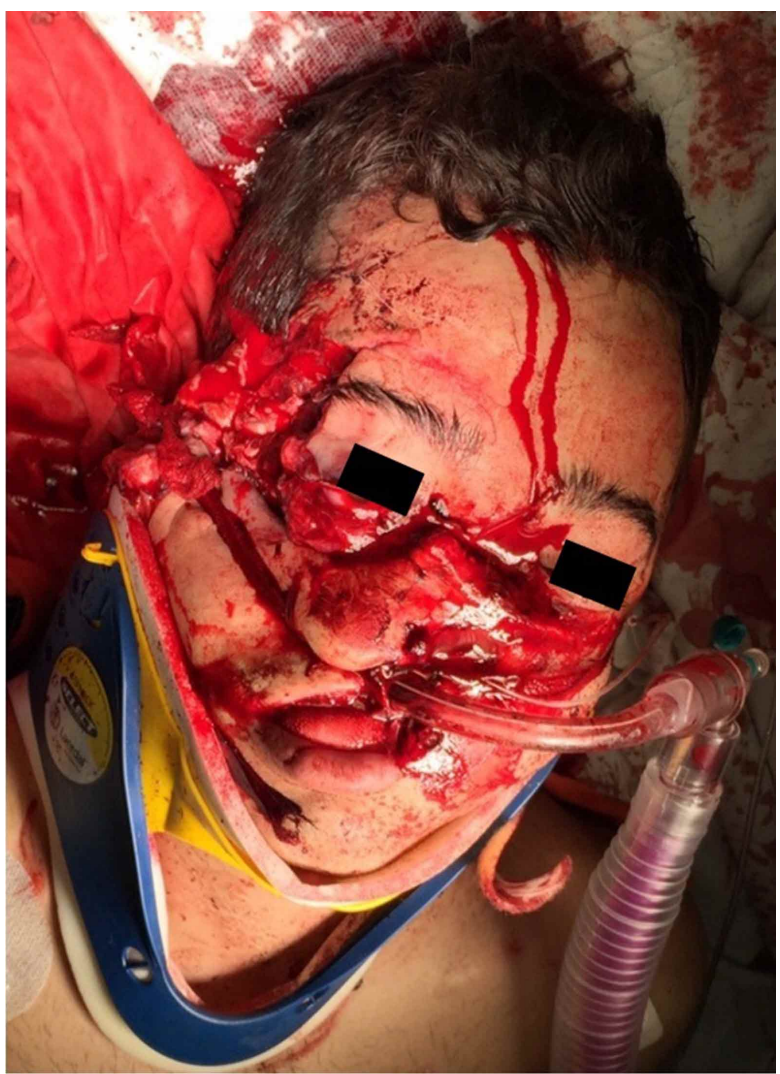

Fig. 1. Paciente con trauma facial severo, intubado para protección de vía aérea. Heridas transfixiantes y a colgajo de hemicara con exposición de huesos faciales. para evaluación primaria de control de la vía aérea y columna cervical, respiración, circulación, valoración del estado neurológico y exposición y control ambiental (ABCDE). Ingresó a la Unidad de Cuidados Intensivos (UCl) para estabilización y observación inicial. Al examen clínico maxilofacial, presentó extensas heridas faciales transfixiantes y a colgajo de hemicara, desplazamiento y exposición de huesos faciales (Fig. 1). La tomografía computarizada (TC) de órbita maxilofacial y mandíbula revela fractura panfacial de tercio inferior y medio, con fractura y desplazamiento de segmento hemimandibular derecho y fracturas conminutas desplazadas del tercio medio facial (Fig. 2).

Luego de la estabilización inicial del paciente, se indicó tratamiento quirúrgico inicial de la fractura panfacial, con el objeto de contener daños, reposicionar estructuras óseas ampliamente desplazadas y lograr síntesis de tejidos blandos comprometidos. Bajo anestesia general, intubación orotraqueal con derivación submental se reducen y fijaron los fragmentos óseos fracturados a placas y tornillos de osteosíntesis, siguiendo la secuencia de reducción desde caudal a cefálico, estabilizando la mandíbula al registro de oclusión más estable que presentaba el paciente, para luego reducir los pilares y arcos del esqueleto facial mandibular, fijando el cuerpo mandibular según los criterios estándar de ostesíntesis a placas y tornillos. Posteriormente, para el tercio medio facial, se realizó la secuencia de lateral hacia medial, desde la estabilización de arcos y pilares laterales hacia los arcos y pilares mediales de la cara. El reposicionamiento del hueso cigomático se logró alineándolo en su unión o sutura con el ala mayor del esfenoides, reconstruyendo de esta forma la pared lateral orbitaria. Una vez reposicionado este hueso se redujeron y fijaron los elementos óseos mediales.
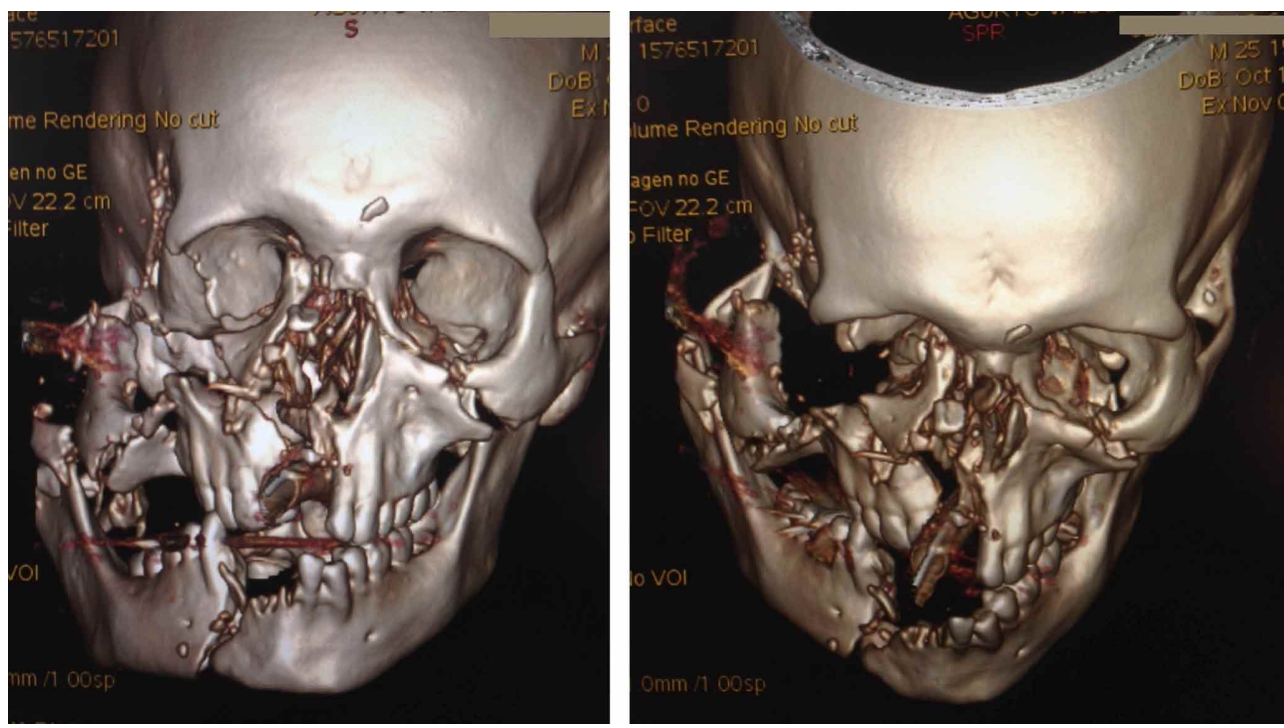

Fig. 2. TC facial reconstrucción 3D, conminución esqueleto óseo facial con desplazamiento severo de estructuras del tercio medio e inferior facial. 
Se suturaron los tejidos blandos por planos (Fig. 3). El paciente evolucionó favorablemente en el post operatorio inmediato (Fig. 4). TC de control se observaron fragmentos óseos en posición anatómica, y reducidos a placas y tornillos de osteosíntesis (Fig. 5). Una vez com- pletada la hospitalización inicial, el paciente fue controlado de forma ambulatoria durante 2 meses, esperando el proceso de cicatrización. Posterior a este período el paciente deberá recibir nuevos procedimientos quirúrgicos para resolver secuelas de su trauma facial.

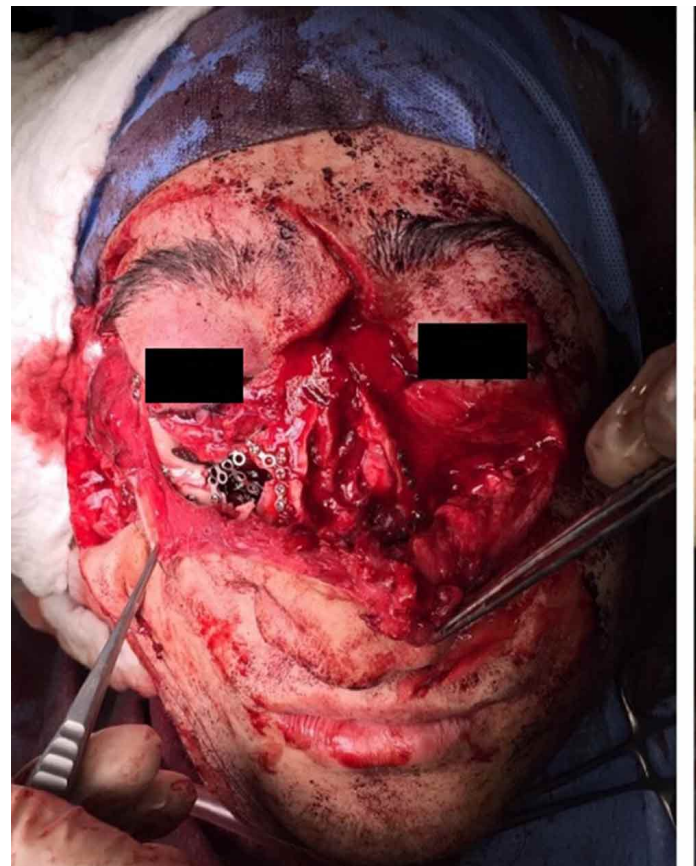

A

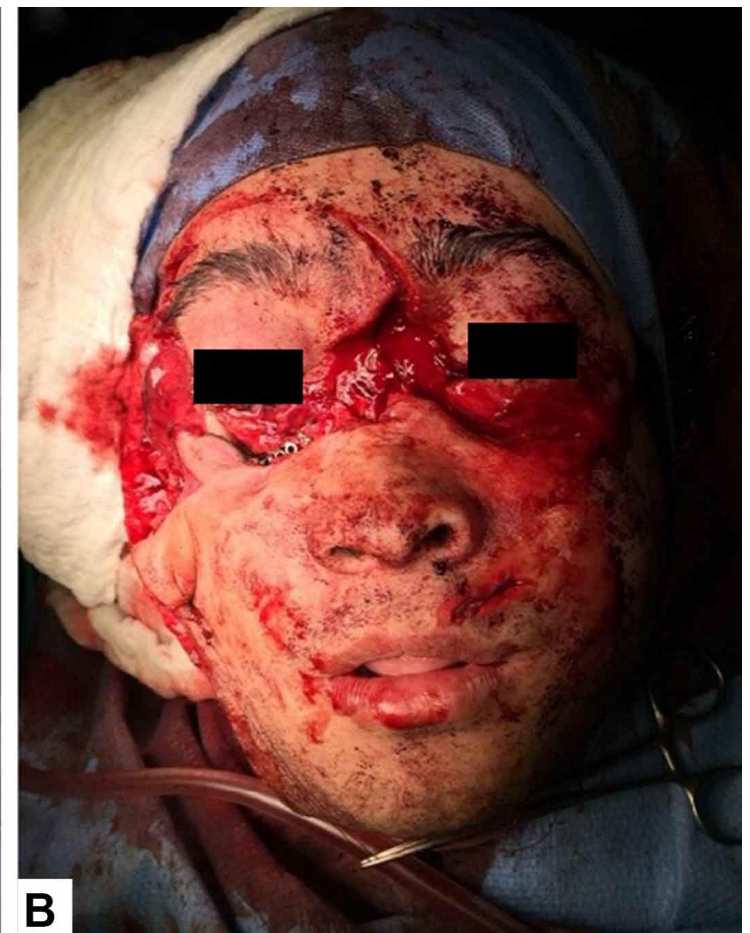

Fig. 3. (A) Reducción y fijación a placas y tornillos de osteosíntesis de fragmentos óseos fracturados tercio medio facial. (B) Reposición de tejidos blandos tercio medio facial.
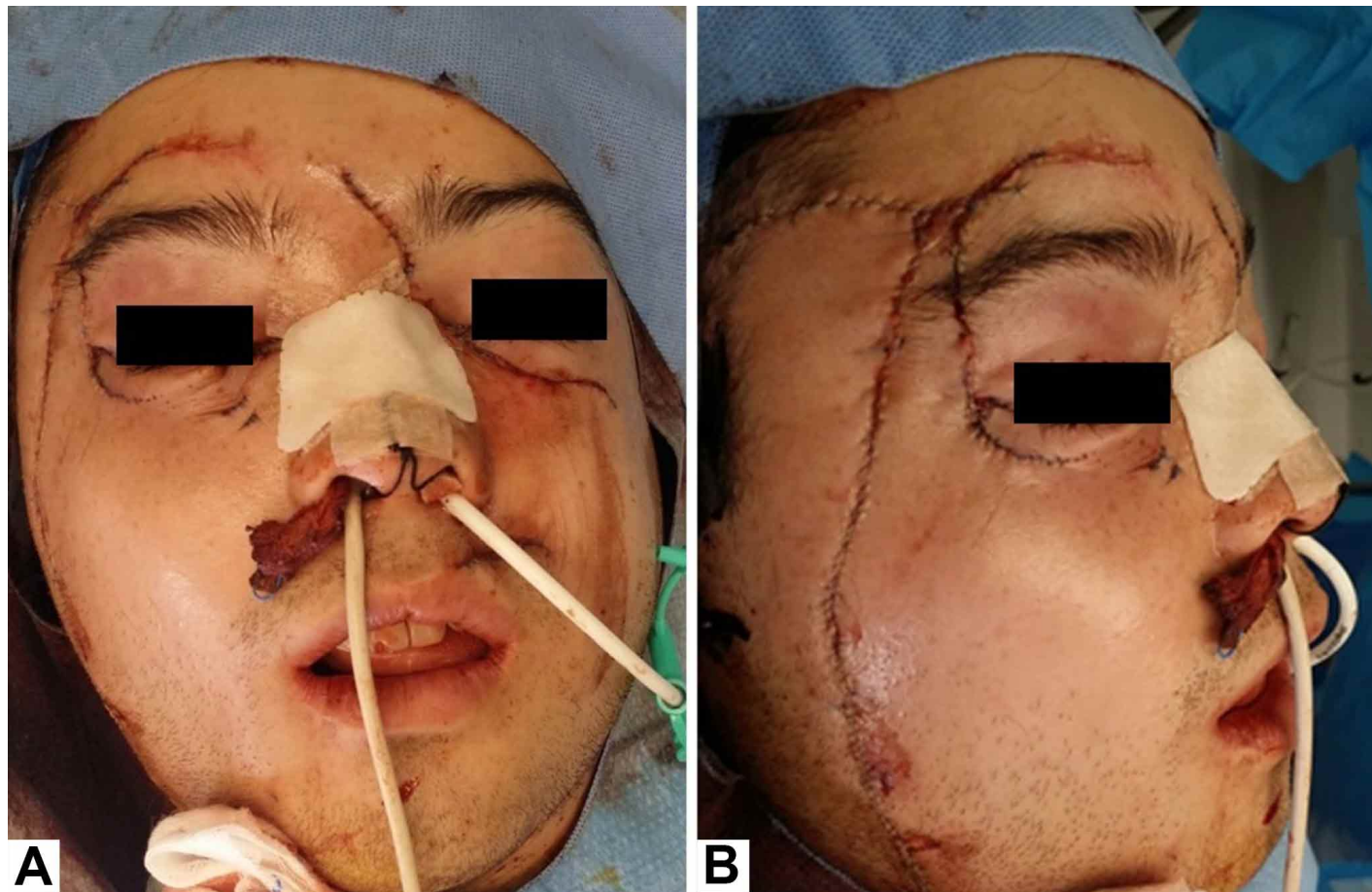

Fig. 4. (A) Imagen frontal de post operatorio inmediato. (B) Imagen lateral de post operatorio inmediato. 


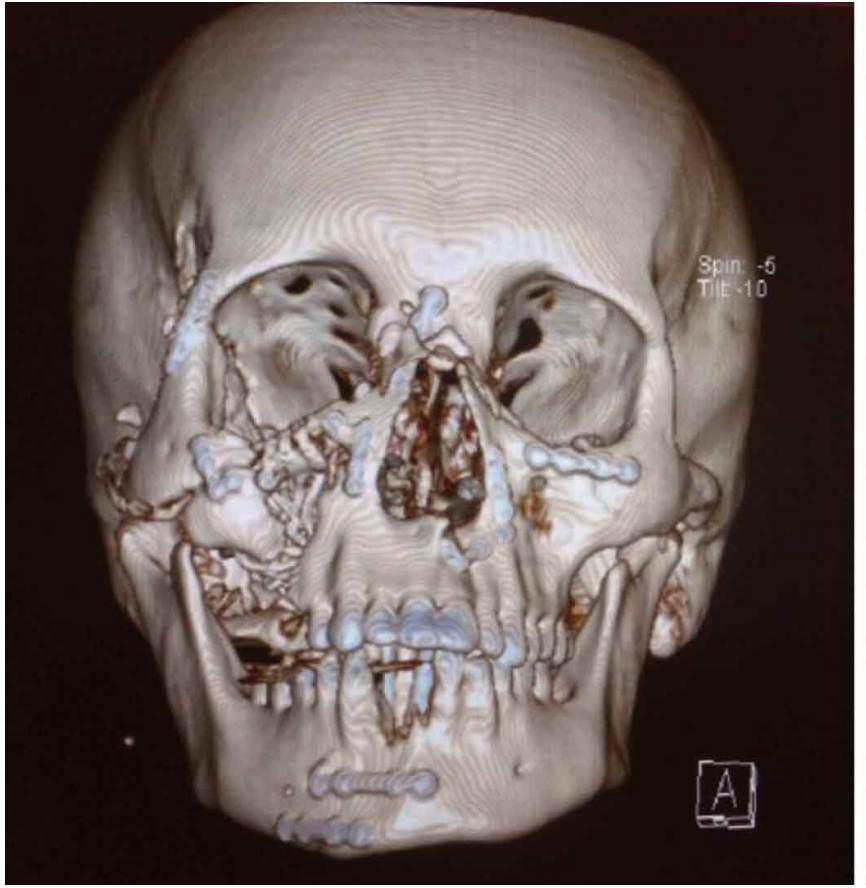

Fig. 5. TC reconstrucción 3D post quirúrgico inmediato.

\section{DISCUSIÓN}

La evaluación y el tratamiento de las fracturas panfaciales puede ser un desafío clínico, ya que involucra simultáneamente huesos de dos o más tercios faciales, en las que el grado de fragmentación dificulta la restauración de la arquitectura original facial (Kim et al.; Wang et al., 2019). Además, suelen estar asociadas a lesiones de columna cervical y/o craneocerebrales y obstrucción de la vía aérea, arriesgando así la vida del paciente (Yang et al.). En una primera instancia el manejo inicial del paciente traumatizado, dado por la Advanced Trauma Life Support (ATLS), se basa en tres principios bien establecidos: Evaluación del ABCDE (Mantenimiento de la vía aérea con protección de la columna cervical, Respiración con ventilación, Circulación con control de hemorragias, Discapacidad y evaluación del estado neurológico, y Exposición/ Entorno); "primum non nocere" (primero, no hacer daño); y el tratamiento de las lesiones que amenazan la vida dentro de la "hora dorada" (Perry, 2008). La primera prioridad es siempre la evaluación de la vía aérea, y simultáneamente la protección de la columna cervical. El compromiso inmediato y retardado de la vía respiratoria en el trauma facial puede surgir como resultado de diversas combinaciones de desplazamiento de tejidos, edemas y hemorragias (Perry \& Morris, 2008). Una vez que

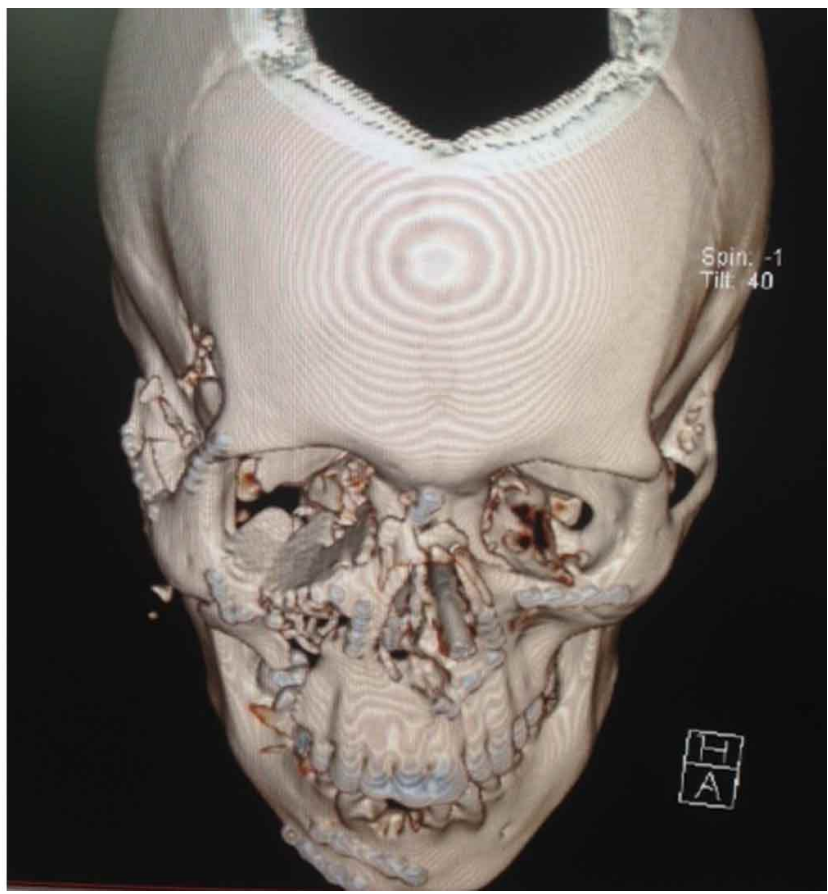

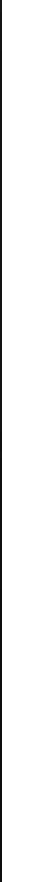


orotraqueal y por otra parte, si existe daño en el tercio medio facial que involucre al complejo naso-etmoidal, para evitar daño a la base de cráneo por intubación involuntaria, es que se contraindicaría la intubación nasotraqueal; es por esto, que la intubación orotraqueal con derivación submental o la traqueostomía serían las formas idóneas de intubación para este tipo de pacientes (Rodrigues et al.).

La intubación realizada en el caso presentado fue orotraqueal con derivación submental, para así facilitar la fijación maxilomandibular, reducción y fijación de los fragmentos óseos fracturados y obtener un acceso a los dos tercios faciales comprometidos sin interferencias (Wang et al.).

El tratamiento exitoso de las fracturas panfaciales requiere la comprensión de los componentes de los patrones de fractura y de la capacidad de relacionarlos anatómicamente con los elementos estables del cráneo. El restablecimiento de la apariencia y la función facial normal sólo es posible si el cirujano puede comprender las tres dimensiones de la anatomía facial: ancho, altura y proyección (Rodrigues et al.; Choi \& Kim).

La reducción adecuada de las fracturas faciales recuperará la funcionalidad alterada por el traumatismo, como la permeabilidad de las vías respiratorias, la restauración de la oclusión, motilidad mandibular y ocular y recuperar la estética facial (Choi \& Kim). Para cumplir con estos objetivos funcionales del tratamiento se debe devolver la continuidad de los arcos y pilares óseos faciales y restaurar los tejidos involucrados en una posición idealmente anatómica (Nwoku et al.).

Para llevar a cabo estos objetivos es importante reconocer las estructuras óseas que componen las tres dimensiones en el espacio: ancho facial central compuesto por complejo naso-órbito-etmoidal (NOE), paladar y arco mandibular (sínfisis); ancho facial lateral compuesto por arco frontal, arco cigomático, eminencia malar y ángulo mandibular. La proyección está determinada por el pilar anterior, arco frontal, arco cigomático, mandíbula (desde el ángulo hasta la sínfisis). Y la altura facial está conformada por el hueso frontal, pilares del tercio medio, y ángulos y cóndilos mandibulares (Curtis \& Horswell, 2013).

Existen 2 secuencias de tratamiento de las fracturas panfaciales, de caudal a cefálico y de lateral a medial (bottom-up, outside-in), o de cefálico a caudal y de medial a lateral (top-down, inside-out), dependiendo del patrón de fractura presente (Choi \& Kim). El ancho o proyección lateral es lo primero que debe ser abordado, la proyección sagital es recíproca a ésta. Mientras que la altura debe ser reconstruida en ambas unidades individualmente (Kim et al.).

El caso presentado fue abordado con la secuencia bottom-up y outside-in, con la reconstrucción primero de la mandíbula y de la oclusión del paciente. La mandíbula determina la altura del tercio inferior de la cara dada por la región del cóndilo y la rama, mientras que el ancho es determinado por el cuerpo mandibular, y la proyección está determinada por la sínfisis. La mandíbula interactúa con el maxilar por oclusión y con la base del cráneo por la articulación temporomandibular, lo que asegura la continuidad tanto del tercio inferior de la cara como de todo el esqueleto facial. Luego se continúa hacia cefálico (de Melo et al., 2013).

Posteriormente se debe realizar la fijación del hueso cigomático, teniendo como principal referencia la correcta alineación en su sutura con el ala mayor del esfenoides, reconstruyendo la pared lateral de la órbita. Al hacer esta maniobra se asegura la correcta posición tridimensional del hueso cigomático, evitando rotaciones o falta de proyección de este hueso, que como estructura ósea del tercio medio facial, constituye el elemento morfológico más importante, debido al volumen de tejido que involucra su estructura. Una vez reposicionado este hueso se reconstruye hacia medial fijando pilares naso-maxilar y el pilar lateral del tercio medio facial, es así, que se sigue la secuencia de lateral a medial (Yang et al.).

Esta secuencia es la más recomendada y estable ya que muestra buenos resultados en cuanto a la restauración de la estructura ósea facial original, permitiendo restaurar el marco y la proyección facial externa. Sin embargo, no siempre es posible utilizar esta secuencia ya que depende del patrón y grado de conminución de las fracturas que presente el paciente.

Por otra parte, los pacientes con fracturas panfaciales, generalmente requieren de múltiples cirugías reconstructivas debido a la severidad del daño estructural y funcional (Wang et al.). El caso presentado describe un tipo de secuencia quirúrgica inicial para abordar fracturas panfaciales, teniendo como objetivo el control de daños, reposición y reconstrucción anatómica y funcional, según el cuadro general que el paciente presentaba. 
Las cirugías posteriores tienen el objetivo de resolver las posibles secuelas morfológicas y/o funcionales que pudiesen presentarse en el post operatorio mediato.

El caso presentado requirió de cirugías complementarias posteriores para resolver secuelas de tejidos blandos.

\section{CONCLUSIÓN}

Las fracturas panfaciales requieren de un manejo inicial que incluye control y protección de la vía aérea y columna cervical, respiración, circulación, valoración del estado neurológico y exposición y control ambiental (ABCDE) para la estabilización del paciente.

El tratamiento inicial maxilofacial para la reparación quirúrgica de las fracturas panfaciales se puede abordar de manera secuencial dependiendo del patrón de fractura del individuo. Esta secuencia permite reconstruir tridimensionalmente de forma funcional y anatómica las estructuras óseas comprometidas. La secuencia bottom-up y outside-in es la más recomendada, ya que permite una reconstrucción facial más confiable y estable desde el punto de vista funcional y anatómico.

MARDONES, M. M.; BRAVO, R. A.; FERNÁNDEZ, T. M. Á.; GUNCKEL, M. R. \& TORRES, M. C. Systematic in the surgical treatment of panfacial fractures applied to a highly complex clinical case. Int. J. Odontostomat., 14(4):590-595, 2020.

ABSTRACT: Panfacial fractures affect bone tissue in two or more thirds of the face simultaneously. The initial management of these patients is to resolve or stabilize the medical-surgical emergencies that they may present. Most of these fractures are associated to trauma of high kinetic energy, which determine the presence of concomitant injuries that can compromise the life of the patient. The surgical treatment of panfacial fractures may include from the initial surgical intervention of damage control, stabilization, reduction and surgical fixation of fractured segments through osteosynthesis, to mediate the reconstruction of tissues affected by the trauma. For the treatment of panfacial fractures there is a surgical approach that aims to contain and/or minimize acute damage and allow for morphological and functional reduction. We report a clinical case of panfacial fracture whose treatment followed this therapeutic approach.

KEY WORDS: panfacial fracture, polytrauma, initial trauma management.

\section{REFERENCIAS BIBLIOGRÁFICAS}

Choi, J. W. \& Kim, M. J. Treatment of panfacial fractures and threedimensional outcome analysis: the occlusion first approach. $J$. Craniofac. Surg., 30(4):1255-8, 2019.

Chukwulebe, S. \& Hogrefe, C. The diagnosis and management of facial bone fractures. Emerg. Med. Clin. North Am., 37(1):13751, 2019.

Curtis, W. \& Horswell, B. B. Panfacial fractures: an approach to management. Oral Maxillofac. Surg. Clin. North Am., 25(4):64960, 2013.

de Melo, W. M.; Sonoda, C. K.; Shinohara, E. H. \& Garcia Jr., I. R. Using the "bottom-up and outside-in" sequence for panfacial fracture management: does it provide a clinical significance? J. Craniofac. Surg., 24(5):e479-81, 2013.

Kim, J.; Choi, J. H.; Chung, Y. K. \& Kim, S. W. Panfacial bone fracture and medial to lateral approach. Arch. Craniofac. Surg., 17(4):181-5, 2016.

Nwoku, A. L.; Al-Balawi, S. A. \& Al-Zahrani, S. A. A modified method of submental oroendotracheal intubation. Saudi Med. J., 23(1):736, 2002.

Perry, M. \& Morris, C. Advanced trauma life support (ATLS) and facial trauma: can one size fit all? Part 2: ATLS, maxillofacial injuries and airway management dilemmas. Int. J. Oral Maxillofac. Surg., 37(4):309-20, 2008.

Perry, M. Advanced Trauma Life Support (ATLS) and facial trauma: can one size fit all? Part 1: dilemmas in the management of the multiply injured patient with coexisting facial injuries. Int. J. Oral Maxillofac. Surg., 37(3):209-14, 2008.

Perry, M.; O'Hare, J. \& Porter, G. Advanced Trauma Life Support (ATLS) and facial trauma: can one size fit all? Part 3: Hypovolaemia and facial injuries in the multiply injured patient. Int. J. Oral Maxillofac. Surg., 37(5):405-14, 2008.

Rodrigues, W. C.; de Melo, W. M.; de Almeida, R. S.; Pardo-Kaba, S. C.; Sonoda, C. K. \& Shinohara, E. H. Submental intubation in cases of panfacial fractures: a retrospective study. Anesth. Prog., 64(3):153-61, 2017.

Wang, L.; Lee, T. S.; Wang, W.; Yi, D. I.; Sokoya, M. \& Ducic, Y. Surgical management of panfacial fractures. Facial Plast. Surg., 35(6):565-77, 2019.

Yang, R.; Zhang, C.; Liu, Y.; Li, Z. \& Li, Z. Why should we start from mandibular fractures in the treatment of panfacial fractures? $\mathrm{J}$. Oral Maxillofac. Surg., 70(6):1386-92, 2012.

Dirección para correspondencia:

Coral Torres Manríquez

Hospital San José

Independencia

Santiago

CHILE

Email: torresm.coral@gmail.com

Recibido : 26-03-2020

Aceptado: $12-05-2020$ 